\title{
Conference Paper \\ Fractional Variational Problems Depending on Fractional Derivatives of Differentiable Functions with Application to Nonlinear Chaotic Systems
}

\author{
Matheus Jatkoske Lazo \\ Instituto de Matemática, Estatística e Física, Universidade Federal do Rio Grande, 96.201-900 Rio Grande, RS, Brazil \\ Correspondence should be addressed to Matheus Jatkoske Lazo; matheuslazo@furg.br
}

Received 15 June 2013; Accepted 14 July 2013

Academic Editors: G. S. F. Frederico, N. Martins, D. F. M. Torres, and A. J. Zaslavski

This Conference Paper is based on a presentation given by Matheus Jatkoske Lazo at "The Cape Verde International Days on Mathematics 2013" held from 22 April 2013 to 25 April 2013 in Praia, Cape Verde.

\begin{abstract}
Copyright (C) 2013 Matheus Jatkoske Lazo. This is an open access article distributed under the Creative Commons Attribution License, which permits unrestricted use, distribution, and reproduction in any medium, provided the original work is properly cited.
\end{abstract}

We formulate a necessary condition for functionals with Lagrangians depending on fractional derivatives of differentiable functions to possess an extremum. The Euler-Lagrange equation we obtained generalizes previously known results in the literature and enables us to construct simple Lagrangians for nonlinear systems. As examples of application, we obtain Lagrangians for some chaotic dynamical systems.

\section{Introduction}

The calculus with fractional derivatives and integrals of noninteger order started more than three centuries ago when Leibniz proposed a derivative of order $1 / 2$ in response to a letter from l'Hôpital [1]. This subject was also considered by several mathematicians as Euler, Fourier, Liouville, Grunwald, Letnikov, Riemann, and others up to nowadays. Although the fractional calculus is almost as old as the usual integer order calculus, only in the last three decades it has gained more attention due to its applications in various fields of science (see [2-7] for a review). Fractional derivatives are generally nonlocal operators and are historically applied to study nonlocal or time-dependent processes, as well as to model phenomena involving coarse-grained and fractal spaces. As an example, applications of fractional calculus in coarse-grained and fractal spaces are found in the framework of anomalous diffusion [8-10] and field theories [11-16].

The fractional calculus of variation was introduced in the context of classical mechanics. Riewe $[17,18]$ showed that a Lagrangian involving fractional time derivatives leads to an equation of motion with nonconservative forces such as friction. It is a remarkable result since frictional and nonconservative forces are beyond the usual macroscopic variational treatment [19] and consequently, beyond the most advanced methods of classical mechanics. Riewe generalized the usual calculus of variations for a Lagrangian depending on fractional derivatives $[17,18]$ in order to deal with linear nonconservative forces. Recently, several approaches have been developed to generalize the least action principle and the Euler-Lagrange equations to include fractional derivatives [20-26].

Despite that the Riewe approach has been successfully applied to study open and/or nonconservative linear systems, it cannot be directly applied to nonlinear open systems. The limitation follows from the fact that, in order to obtain a final equation of motion containing only integer order derivatives, the Lagrangian should contain only quadratic terms depending on fractional derivatives. In the present work we formulated a generalization of Riewe fractional action principle by taking advantage of a so-called practical limitation of fractional derivatives, namely, the absence of a simple chain and Leibniz's rules. 
As examples, we applied our generalized fractional variational principle to some nonlinear chaotic third-order dynamical systems, so-called jerk dynamical systems because the derivative of the acceleration with respect to time is referred to as the jerk [27]. These systems are important because they are the simplest ever one-dimensional autonomous ordinary differential equations which display dynamical behaviors including chaotic solutions [28-35]. It is important to mention that jerk dynamical systems describe several phenomena in physics, engineering, and biology, such as electrical circuits, mechanical oscillators, laser physics, and biological systems [28-35].

\section{The Riemann-Liouville and Caputo Fractional Calculus}

The fractional calculus of derivative and integration of noninteger orders started more than three centuries ago with l'Hôpital and Leibniz when the derivative of order $1 / 2$ was suggested [1]. This subject was also considered by several mathematicians as Euler, Laplace, Liouville, Grunwald, Letnikov, Riemann and others up to nowadays. Although the fractional calculus is almost as old as the usual integer order calculus, only in the last three decades it has gained more attention due to its applications in various fields of science, engineering, economics, biomechanics, and so forth (see $[2,3,5,6]$ for a review). Actually, there are several definitions of fractional order derivatives. These definitions include the Riemann-Liouville, Caputo, Riesz, Weyl, and Grunwald-Letnikov (see [1-7] for a review). In this section we review some definitions and properties of the RiemannLiouville and Caputo fractional calculi.

Despite haing many different approaches to fractional calculus, several known formulations are somehow connected with the analytical continuation of Cauchy formula for $n$-fold integration:

$$
\begin{aligned}
& \int_{a}^{t} x(\tilde{t})(d \tilde{t})^{n} \\
& \quad=\int_{a}^{t} \int_{a}^{t_{n}} \int_{a}^{t_{n-1}} \cdots \int_{a}^{t_{3}} \int_{a}^{t_{2}} x\left(t_{1}\right) d t_{1} d t_{2} \cdots d t_{n-1} d t_{n} \\
& \quad=\frac{1}{\Gamma(n)} \int_{a}^{t} \frac{x(u)}{(t-u)^{1-n}} d u \quad(n \in \mathbb{N}),
\end{aligned}
$$

where $\Gamma$ is the Euler gamma function. The proof of Cauchy formula can be found in several textbooks (e.g., it can be found in [1]). The analytical continuation of (1) gives us a definition for an integration of noninteger (or fractional) order. This fractional order integration is the building block of the Riemann-Liouville and Caputo calculi, the two most popular formulations of fractional calculus, as well as several other approaches to fractional calculus [1-7]. The fractional integration obtained from (1) is historically called RiemannLiouville left and right fractional integrals.
Definition 1. Let $\alpha \in \mathbb{R}_{+}$. The operators ${ }_{a} J_{t}^{\alpha}$ and ${ }_{t} J_{b}^{\alpha}$ defined on $L_{1}[a, b]$ by

$$
\begin{array}{ll}
{ }_{a} J_{t}^{\alpha} x(t)=\frac{1}{\Gamma(\alpha)} \int_{a}^{t} \frac{x(u)}{(t-u)^{1-\alpha}} d u & \left(\alpha \in \mathbb{R}_{+}\right), \\
{ }_{t} J_{b}^{\alpha} x(t)=\frac{1}{\Gamma(\alpha)} \int_{t}^{b} \frac{x(u)}{(u-t)^{1-\alpha}} d u & \left(\alpha \in \mathbb{R}_{+}\right),
\end{array}
$$

with $a<b$ and $a, b \in \mathbb{R}$, are called left and right fractional Riemann-Liouville integrals of order $\alpha \in \mathbb{R}$, respectively.

For integer $\alpha$ the fractional Riemann-Liouville integrals (2) coincide with the usual integer order $n$-fold integration (1). Moreover, from definitions (2) it is easy to see that the Riemann-Liouville fractional integrals converge for any integrable function $x$ if $\alpha>1$. Furthermore, it is possible to prove the convergence of (2) for $x \in L_{1}[a, b]$ even when $0<\alpha<1[4]$.

The integration operators ${ }_{a} J_{t}^{\alpha}$ and ${ }_{t} J_{b}^{\alpha}$ play a fundamental role in the definition of fractional Riemann-Liouville and Caputo calculi. In order to define the Riemann-Liouville derivatives, we recall that for positive integers $n>m$ it follows the identity $D_{t}^{m} x(t)=D_{t a}^{n} J_{t}^{n-m} x(t)$, where $D_{t}^{m}$ is an ordinary derivative of integer order $m$.

Definition 2 (Riemann-Liouville). The left and the right Riemann-Liouville fractional derivatives of order $\alpha>0(\alpha \in$ $\mathbb{R})$ are defined, respectively, by ${ }_{a} D_{t}^{\alpha} x(t):=D_{t}^{n} J_{t}^{n-\alpha} x(t)$ and ${ }_{t} D_{b}^{\alpha} x(t):=(-1)^{n} D_{t}^{n} J_{b}^{n-\alpha} x(t)$ with $n=[\alpha]+1$, namely,

$$
\begin{aligned}
& { }_{a} D_{t}^{\alpha} x(t)=\frac{1}{\Gamma(n-\alpha)} \frac{d^{n}}{d t^{n}} \int_{a}^{t} \frac{x(u)}{(t-u)^{1+\alpha-n}} d u, \\
& { }_{t} D_{b}^{\alpha} x(t)=\frac{(-1)^{n}}{\Gamma(n-\alpha)} \frac{d^{n}}{d t^{n}} \int_{t}^{b} \frac{x(u)}{(u-t)^{1+\alpha-n}} d u,
\end{aligned}
$$

where $d^{n} / d t^{n}$ stands for ordinary derivatives of integer order $n$.

On the other hand, the Caputo fractional derivatives are defined by inverting the order between derivatives and integrations.

Definition 3 (Caputo). The left and the right Caputo fractional derivatives of order $\alpha \in \mathbb{R}_{+}$are defined, respectively, by ${ }_{a}^{C} D_{t}^{\alpha} x(t):={ }_{a} J_{t}^{n-\alpha} D_{t}^{n} x(t)$ and ${ }_{t}^{C} D_{b}^{\alpha} x(t):=(-1)_{t}^{n} J_{b}^{n-\alpha} D_{t}^{n} x(t)$ with $n=[\alpha]+1$; that is,

$$
\begin{aligned}
& { }_{a}^{C} D_{t}^{\alpha} x(t):=\frac{1}{\Gamma(n-\alpha)} \int_{a}^{t} \frac{x^{(n)}(u)}{(t-u)^{1+\alpha-n}} d u, \\
& { }_{t}^{C} D_{b}^{\alpha} x(t):=\frac{(-1)^{n}}{\Gamma(n-\alpha)} \int_{t}^{b} \frac{x^{(n)}(u)}{(u-t)^{1+\alpha-n}} d u,
\end{aligned}
$$

where $a \leq t \leq b$ and $x^{(n)}(u)=d^{n} x(u) / d u^{n} \in L_{1}([a, b])$ is the ordinary derivative of integer order $n$.

An important consequence of definitions (3)-(6) is that the Riemann-Liouville and Caputo fractional derivatives are 
nonlocal operators. The left (right) differintegration operator (3) and (5) ((4) and (6)) depends on the values of the function at left (right) of $t$, that is, $a \leq u \leq t(t \leq u \leq b)$. On the other hand, it is important to note that when $\alpha$ is an integer, the Riemann-Liouville fractional derivatives (3) and (4) and reduced to ordinary derivatives of order $\alpha$. On the other hand, in that case, the Caputo derivatives (5) and (6) differ from integer order ones by a polynomial of order $\alpha-1[3,4]$.

It is important to remark, for the purpose of this work, that the fractional derivatives (3)-(6) do not satisfy a simple generalization of the chain and Leibniz's rules of classical derivatives [1-7]. In other words, generally we have

$$
\begin{gathered}
{ }_{a}^{C} D_{t}^{\alpha}[x(t) y(t)] \neq y(t){ }_{a}^{C} D_{t}^{\alpha} x(t)+x(t){ }_{a}^{C} D_{t}^{\alpha} y(t), \\
{ }_{a}^{C} D_{t}^{\alpha} y(x(t)) \neq\left.{ }_{a}^{C} D_{u}^{\alpha} y(u)\right|_{u=x}{ }_{a}^{C} D_{t}^{\alpha} x(t) .
\end{gathered}
$$

The absence of a simple chain and Leibniz's rules is commonly considered a practical limitation of the fractional derivatives (3)-(6). However, in the present work we take advantage of this limitation in order to formulate a generalized Lagrangians for nonlinear systems.

In addition to the definitions (3)-(6), we make use of the following property in order to obtain a fractional generalization of the Euler-Lagrange condition.

Theorem 4 (integration by parts-see, e.g., [7]). Let $0<\alpha<$ 1 and let $x$ be a differentiable function in $[a, b]$ with $x(a)=$ $x(b)=0$. For any function $y \in L_{1}([a, b])$ one has

$$
\begin{aligned}
& \int_{a}^{b} y(t){ }_{a}^{C} D_{t}^{\alpha} x(t) d t=\int_{a}^{b} x(t){ }_{t} D_{b}^{\alpha} y(t) d t, \\
& \int_{a}^{b} y(t){ }_{t}^{C} D_{b}^{\alpha} x(t) d t=\int_{a}^{b} x(t){ }_{a} D_{t}^{\alpha} y(t) d t .
\end{aligned}
$$

It is important to notice that the formulas of integration by parts (8) relate Caputo left (right) derivatives to RiemannLiouville right (left) derivatives.

Finally, in order to obtain the equation of motion for our examples, we are going to use the following two relations:

$$
\begin{aligned}
& i_{a}^{C} D_{t}^{1 / 2} x(t)={ }_{t}^{C} D_{b}^{1 / 2} x(t) \quad \text { in the limit } a \longrightarrow b, \\
&{ }_{t} D_{b}^{1 / 2}{ }_{t}^{C} D_{b}^{1 / 2} x(t)=\frac{d}{d t}{ }_{t} J_{b}^{1 / 2}{ }_{t} J_{b}^{1 / 2} \frac{d}{d t} x(t) \\
&=\frac{d}{d t}{ }_{t} J_{b}^{1} \frac{d}{d t} x(t)=\frac{d}{d t} x(t) .
\end{aligned}
$$

The proof of (9) can be found in [36], and (10) follows from the general semigroup property ${ }_{t} J_{b}^{\alpha}{ }_{t} J_{b}^{\beta}={ }_{t} J_{b}^{\alpha+\beta}$ (see, e.g., [4, 7]).

\section{A Generalized Fractional Lagrangian}

In classical calculus of variations it is of no conceptual and practical importance to deal with Lagrangian functions depending on derivatives of nonlinear functions of the unknown function $x$. This is due to the fact that in these cases we can always rewrite the Lagrangian $L$ as a usual Lagrangian $\widetilde{L}$ by applying the chain's rules. As, for example, for a differentiable function $f$ we can rewrote $L(t, x,(d / d t) f(x))=L\left(t, x,\left.(d / d u) f(u)\right|_{u=x} \dot{x}\right)=\widetilde{L}(t, x, \dot{x})$, where $d x / d t=\dot{x}$. However, this simplification for the fractional calculus of variation is not possible due to the absence of a simple chain's rule for fractional derivatives. It is just this apparent limitation of fractional derivatives that opens the very interesting possibility to investigate new kinds of Lagrangians suitable to study nonlinear systems. In the present work we investigate for the first time these kinds of Lagrangians and we apply them to construct Lagrangians for some Jerk nonlinear dynamical system.

Our main result is the following theorem.

Theorem 5. Let $f, g: \mathbb{R} \rightarrow \mathbb{R}$ be differentiable functions, and $S$ an action of the form

$$
S=\int_{a}^{b} L\left(t, x, \dot{x},{ }_{a}^{C} D_{t}^{\alpha} f(x),{ }_{a}^{C} D_{t}^{\alpha} g(\dot{x})\right) d t,
$$

where ${ }_{a}^{C} D_{t}^{\alpha}$ is a Caputo fractional derivatives of order $0<\alpha<$ 1 , and the function $x$ satisfies the fixed boundary conditions $x(a)=x_{a}, x(b)=x_{b}, \dot{x}(a)=\dot{x}_{a}$, and $\dot{x}(b)=\dot{x}_{b}$. Also let $L \in C^{2}[a, b] \times \mathbb{R}^{4}$. Then, the necessary condition for $S$ to possess an extremum at $x$ is that the function $x$ fulfills the following fractional Euler-Lagrange equation:

$$
\frac{\partial L}{\partial x}-\frac{d}{d t} \frac{\partial L}{\partial \dot{x}}+\frac{d f}{d x}{ }_{t} D_{b}^{\alpha} \frac{\partial L}{\partial\left({ }_{a}^{C} D_{t}^{\alpha} f\right)}-\frac{d}{d t}\left(\frac{d g}{d \dot{x}} D^{\alpha} D_{b}^{\alpha} \frac{\partial L}{\partial\left({ }_{a}^{C} D_{t}^{\alpha} g\right)}\right)=0 .
$$

Proof. In order to develop the necessary conditions for the extremum of the action (11), we define a family of functions $x$ (weak variations)

$$
x=x^{*}+\varepsilon \eta
$$

where $x^{*}$ is the desired real function that satisfy the extremum of (11), $\varepsilon \in \mathbb{R}$ is a constant, and the function $\eta$ defined in $[a, b]$ satisfies the boundary conditions

$$
\eta(a)=\eta(b)=0, \quad \dot{\eta}(a)=\dot{\eta}(b)=0 .
$$

The condition for the extremum is obtained when the first Gâteaux variation is zero:

$$
\begin{aligned}
& \delta S=\lim _{\varepsilon \rightarrow 0} \frac{S\left[x^{*}+\varepsilon \eta\right]-S\left[x^{*}\right]}{\varepsilon} \\
&=\int_{a}^{b}\left[\eta \frac{\partial L}{\partial x^{*}}+\dot{\eta} \frac{\partial L}{\partial\left(\dot{x}^{*}\right)}+\left({ }_{a}^{C} D_{t}^{\alpha} \eta \frac{d f}{d x^{*}}\right) \frac{\partial L}{\partial\left({ }_{a}^{C} D_{t}^{\alpha} f\right)}\right. \\
&\left.+\left({ }_{a}^{C} D_{t}^{\alpha} \dot{\eta} \frac{d g}{d \dot{x}^{*}}\right) \frac{\partial L}{\partial\left({ }_{a}^{C} D_{t}^{\alpha} g\right)}\right] d t=0 .
\end{aligned}
$$


Since the function $\eta$ satisfies both $\eta(a)=\eta(b)=0$ and $\dot{\eta}(a)=$ $\dot{\eta}(b)=0$ boundary conditions (14), we can use the fractional integration by parts (8) in (15), obtaining

$$
\begin{aligned}
& \delta S=\int_{a}^{b}\left[\eta \frac{\partial L}{\partial x^{*}}+\dot{\eta} \frac{\partial L}{\partial\left(\dot{x}^{*}\right)}+\eta \frac{d f}{d x^{*}}{ }_{t} D_{b}^{\alpha} \frac{\partial L}{\partial\left({ }_{a}^{C} D_{t}^{\alpha} f\right)}\right. \\
&\left.+\quad \dot{\eta} \frac{d g}{d \dot{x}^{*}}{ }_{t} D_{b}^{\alpha} \frac{\partial L}{\partial\left({ }_{a}^{C} D_{t}^{\alpha} g\right)}\right] d t \\
&=\int_{a}^{b} \eta\left[\frac{\partial L}{\partial x^{*}}-\frac{d}{d t} \frac{\partial L}{\partial\left(\dot{x}^{*}\right)}+\frac{d f}{d x^{*}}{ }_{t} D_{b}^{\alpha} \frac{\partial L}{\partial\left({ }_{a}^{C} D_{t}^{\alpha} f\right)}\right. \\
&\left.-\frac{d}{d t}\left(\frac{d g}{d \dot{x}^{*}}{ }_{t} D_{b}^{\alpha} \frac{\partial L}{\partial\left({ }_{a}^{C} D_{t}^{\alpha} g\right)}\right)\right] d t=0,
\end{aligned}
$$

where an additional usual integration by parts was performed in the terms containing $\dot{\eta}$. Finally, by using the fundamental lemma of the calculus of variations, we obtain the fractional Euler-Lagrange equations (12).

It is important to notice that our theorem can be easily extended for Lagrangians depending on left Caputo derivatives, and Riemann-Liouville fractional derivatives. Actually, it is also easy to generalize in order to include a nonlinear function $g\left({ }_{a}^{C} D_{t}^{\alpha} x\right)$ instead of $g(\dot{x})$. Finally, it is important to mention that our theorem generalizes $[17,18]$ and the more general formulation proposed in [20], as well as the Lagrangian formulation for higher order linear open systems [37] (for a review in recent advances in calculus of variations with fractional derivatives see [26]).

Remark 6. For $f(x)=g(\dot{x})=0$ our condition (12), reduced to the ordinary Euler-Lagrange equation, and the boundary conditions $x(a)=x_{a}, x(b)=x_{b}$ and $\dot{x}(a)=\dot{x}_{a}, \dot{x}(b)=\dot{x}_{b}$ are defined by only two arbitrary parameters. Note that for this particular case the Euler-Lagrange equation is a second-order ordinary differential equation whose solution $x(t)$ is fixed by two parameters. For example, by imposing the conditions $x(a)=x_{a}$ and $x(b)=x_{b}$ the solution $x(t)$ is fixed and, consequently, the numbers $\dot{x}_{a}$ and $\dot{x}_{b}$ are automatically fixed as functions of $x_{a}$ and $x_{b}$.

\section{Lagrangian for Nonlinear Chaotic Jerk Systems}

As an example for application of our generalized EulerLagrange equation (12), in this section we obtained Lagrangians for some jerk systems. The first example is the simplest one-dimensional family of jerk systems that displays chaotic solutions [27-35]:

$$
\dddot{x}+A \ddot{x}+\dot{x}=G(x),
$$

where $A$ is a system parameter and $G(x)$ is a nonlinear function containing one nonlinearity, one system parameter and a constant term. A Lagrangian for this jerk system is given by

$$
\begin{aligned}
L\left(x,{ }_{t}^{C} D_{b}^{1 / 2} x,{ }_{t}^{C} D_{b}^{1 / 2} \dot{x}\right)= & -\frac{i}{2}\left({ }_{a}^{C} D_{t}^{1 / 2} \dot{x}\right)^{2}-\frac{A}{2}(\dot{x})^{2} \\
& +\frac{i}{2}\left({ }_{a}^{C} D_{t}^{1 / 2} x\right)^{2}-\int G(x) d x
\end{aligned}
$$

In order to show that (18) gives us (17), we insert (18) into our generalized Euler-Lagrange equation (12), obtaining

$$
i \frac{d}{d t}\left({ }_{t}^{C} D_{b}^{1 / 2}{ }_{a}^{C} D_{t}^{1 / 2}\right) \dot{x}+A \ddot{x}+i\left({ }_{t}^{C} D_{b}^{1 / 2}{ }_{a}^{C} D_{t}^{1 / 2}\right) x=G(x),
$$

and we follow the procedure introduced in $[17,18]$ by taking the limit $a \rightarrow b$. Taking the limit in (19) and using (9) and (10) we get (17).

The Lagrangian (18) is equivalent to the introduced by us in [37]. However, it is important to stress that (17) is the only chaotic jerk system containing nonlinearity depending only on $x$ [27-35]. For jerk systems with more complex nonlinearities, as for example, $x \dot{x}, \dot{x}^{2}$, and $x \ddot{x}$, it is not possible to formulate a simple Lagrangian, depending only on $x$ and its derivatives, by using classical calculus of variation or previous formulations including fractional derivatives. Using our Euler-Lagrange equation (12) we can formulate, by the first time, a Lagrangian for these jerk systems [27-35]:

$$
\begin{aligned}
& L=-\frac{i}{2}\left({ }_{a}^{C} D_{t}^{1 / 2} \dot{x}\right)^{2}-\frac{A}{2}(\dot{x})^{2}+\frac{i}{2}\left({ }_{a}^{C} D_{t}^{1 / 2} x^{2}\right){ }_{a}^{C} D_{t}^{1 / 2} \dot{x} \\
& +\frac{x^{2}}{2} \Longrightarrow \ddot{x}+A \ddot{x}-\dot{x}^{2}+x=0 \text {, } \\
& L=-\frac{i}{2}\left({ }_{a}^{C} D_{t}^{1 / 2} \dot{x}\right)^{2}-\frac{A}{2}(\dot{x})^{2}-\frac{i}{2}\left({ }_{a}^{C} D_{t}^{1 / 2} x^{2}\right){ }_{a}^{C} D_{t}^{1 / 2} x \\
& +\frac{x^{2}}{2} \Longrightarrow \ddot{x}+A \ddot{x}-x \dot{x}+x=0 \text {, } \\
& L=-\frac{i}{2}\left({ }_{a}^{C} D_{t}^{1 / 2} \dot{x}\right)^{2}-\frac{A}{2} x(\dot{x})^{2}+i \frac{A+2}{4}\left({ }_{a}^{C} D_{t}^{1 / 2} x^{2}\right){ }_{a}^{C} D_{t}^{1 / 2} \dot{x} \\
& +\frac{x^{2}}{2} \Longrightarrow \ddot{x}+A x \ddot{x}-\dot{x}^{2}+x=0 \text {. }
\end{aligned}
$$

\section{Conclusions}

In the present work we obtained an Euler-Lagrange equation for Lagrangians depending on fractional derivatives of nonlinear functions of the unknown function $x$. Our formulation enables us to obtain Lagrangians for nonlinear open and dissipative systems, and consequently, it enables us to use the most advanced methods of classical mechanics to study these systems. As examples of applications, we obtained a Lagrangian for some chaotic jerk dynamical system. 


\section{Acknowledgments}

The author is grateful to the Brazilian foundations FAPERGS, $\mathrm{CNPq}$, and Capes for financial support.

\section{References}

[1] K. B. Oldham and J. Spanier, The Fractional Calculus, Academic Press, New York, NY, USA, 1974.

[2] J. Sabatier, O. P. Agrawal, and J. A. Tenreiro Machado, Eds., Advances in Fractional Calculus: Theoretical Developments and Applications in Physics and Engineering, Springer, Dordrecht, The Netherlands, 2007.

[3] A. A. Kilbas, H. M. Srivastava, and J. J. Trujillo, Theory and Applications of Fractional Differential Equations, Elsevier, Amsterdam, The Netherlands, 2006.

[4] K. Diethelm, The Analysis of Fractional Differential Equations: An Application-Oriented Exposition Using Differential Operators of Caputo Type, vol. 2004, Springer, Berlin, Germany, 2010.

[5] R. Hilfer, Ed., Applications of Fractional Calculus in Physics, World Scientific Publishing, Singapore, 2000.

[6] R. L. Magin, Fractional Calculus in Bioengineering, Begell House Publisher, 2006.

[7] S. G. Samko, A. A. Kilbas, and O. I. Marichev, Fractional Integrals and Derivatives. Theory and Applications, Gordon and Breach Science Publishers, Yverdon, Switzerland, 1993.

[8] R. Metzler and J. Klafter, "The random walk's guide to anomalous diffusion: a fractional dynamics approach," Physics Report, vol. 339, no. 1, pp. 1-77, 2000.

[9] R. Metzler and J. Klafter, "The restaurant at the end of the random walk: recent developments in the description of anomalous transport by fractional dynamics," Journal of Physics A, vol. 37, no. 31, pp. R161-R208, 2004.

[10] R. Klages, G. Radons, and I. M. Sokolov, Eds., Anomalous Transport: Foundations and Applications, Wiley-VCH, Weinheim, Germany, 2007.

[11] V. E. Tarasov, "Electromagnetic fields on fractals," Modern Physics Letters A, vol. 21, no. 20, pp. 1587-1600, 2006.

[12] M. J. Lazo, "Gauge invariant fractional electromagnetic fields," Physics Letters A, vol. 375, no. 41, pp. 3541-3546, 2011.

[13] E. Baskin and A. Iomin, "Electrostatics in fractal geometry: fractional calculus approach," Chaos, Solitons and Fractals, vol. 44, no. 4-5, pp. 335-341, 2011.

[14] G. Calcagni, "Quantum field theory, gravity and cosmology in a fractal universe," Journal of High Energy Physics, vol. 2010, no. 3, article 120, 2010.

[15] G. Calcagni, "Geometry and field theory in multi-fractional spacetime," Journal of High Energy Physics, vol. 2012, no. 1, article 065, 2012.

[16] S. I. Vacaru, "Fractional dynamics from Einstein gravity, general solutions, and black holes," International Journal of Theoretical Physics, vol. 51, no. 5, pp. 1338-1359, 2012.

[17] F. Riewe, "Nonconservative Lagrangian and Hamiltonian mechanics," Physical Review E, vol. 53, no. 2, pp. 1890-1899, 1996.

[18] F. Riewe, "Mechanics with fractional derivatives," Physical Review E, vol. 55, no. 3, pp. 3581-3592, 1997.

[19] P. S. Bauer, "Dissipative dynamical systems: I," Proceedings of the National Academy of Sciences, vol. 17, pp. 311-314, 1931.
[20] O. P. Agrawal, "Formulation of Euler-Lagrange equations for fractional variational problems," Journal of Mathematical Analysis and Applications, vol. 272, no. 1, pp. 368-379, 2002.

[21] D. Baleanu and O. P. Agrawal, "Fractional Hamilton formalism within Caputo's derivative," Czechoslovak Journal of Physics, vol. 56, no. 10-11, pp. 1087-1092, 2006.

[22] J. Cresson, "Fractional embedding of differential operators and Lagrangian systems," Journal of Mathematical Physics, vol. 48, no. 3, Article ID 033504, 2007.

[23] R. Almeida, S. Pooseh, and D. F. M. Torres, "Fractional variational problems depending on indefinite integrals," Nonlinear Analysis, Theory, Methods and Applications, vol. 75, no. 3, pp. 1009-1025, 2012.

[24] R. Almeida and D. F. M. Torres, "Necessary and sufficient conditions for the fractional calculus of variations with Caputo derivatives," Communications in Nonlinear Science and Numerical Simulation, vol. 16, no. 3, pp. 1490-1500, 2011.

[25] T. Odzijewicz, A. B. Malinowska, and D. F. M. Torres, "Fractional variational calculus with classical and combined Caputo derivatives," Nonlinear Analysis, Theory, Methods and Applications, vol. 75, no. 3, pp. 1507-1515, 2012.

[26] A. B. Malinowska and D. F. M. Torres, Introduction to the Fractional Calculus of Variations, Imperial College Press, London, UK, 2012.

[27] S. H. Schot, "Jerk: the time rate of change of acceleration," American Journal of Physics, vol. 46, pp. 1090-1094, 1978.

[28] J. C. Sprott, Elegant Chaos, World Scientific Publishing Co, 2010.

[29] H. P. W. Gottlieb, "What is the simplest jerk function that gives chaos?" American Journal of Physics, vol. 64, p. 525, 1996.

[30] J. C. Sprott, "Some simple chaotic jerk functions," American Journal of Physics, vol. 65, no. 6, pp. 537-543, 1997.

[31] S. J. Linz, "Nonlinear dynamical models and jerky motion," American Journal of Physics, vol. 65, no. 6, pp. 523-526, 1997.

[32] J. C. Sprott, "Simplest dissipative chaotic flow," Physics Letters A, vol. 228, no. 4-5, pp. 271-274, 1997.

[33] S. J. Linz and J. C. Sprott, "Elementary chaotic flow," Physics Letters A, vol. 259, no. 3-4, pp. 240-245, 1999.

[34] J. C. Sprott, "A new class of chaotic circuit," Physics Letters A, vol. 266, no. 1, pp. 19-23, 2000.

[35] V. Patidar and K. K. Sud, "Identical synchronization in chaotic jerk dynamical systems," Electronic Journal of Theoretical Physics, vol. 3, no. 11, pp. 33-70, 2006.

[36] K. M. Tarawneh, E. M. Rabei, and H. B. Ghassib, "Lagrangian and Hamiltonian formulations of the damped harmonic oscillator using Caputo fractional derivative," Journal of Dynamical Systems and Geometric Theories, vol. 8, no. 1, pp. 59-70, 2010.

[37] M. J. Lazo and C. E. Krumreich, "Lagrangian formulation for open and dissipative systems with higher-order derivatives," submitted to Physica Scripta. 


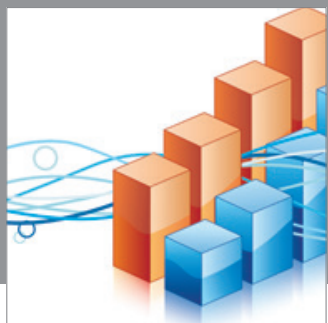

Advances in

Operations Research

mansans

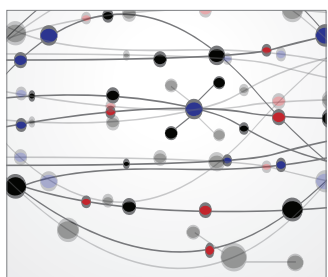

The Scientific World Journal
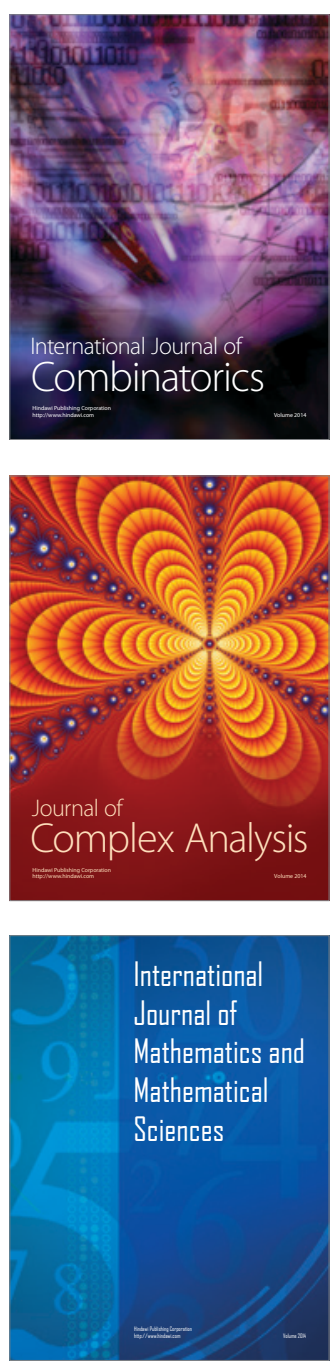
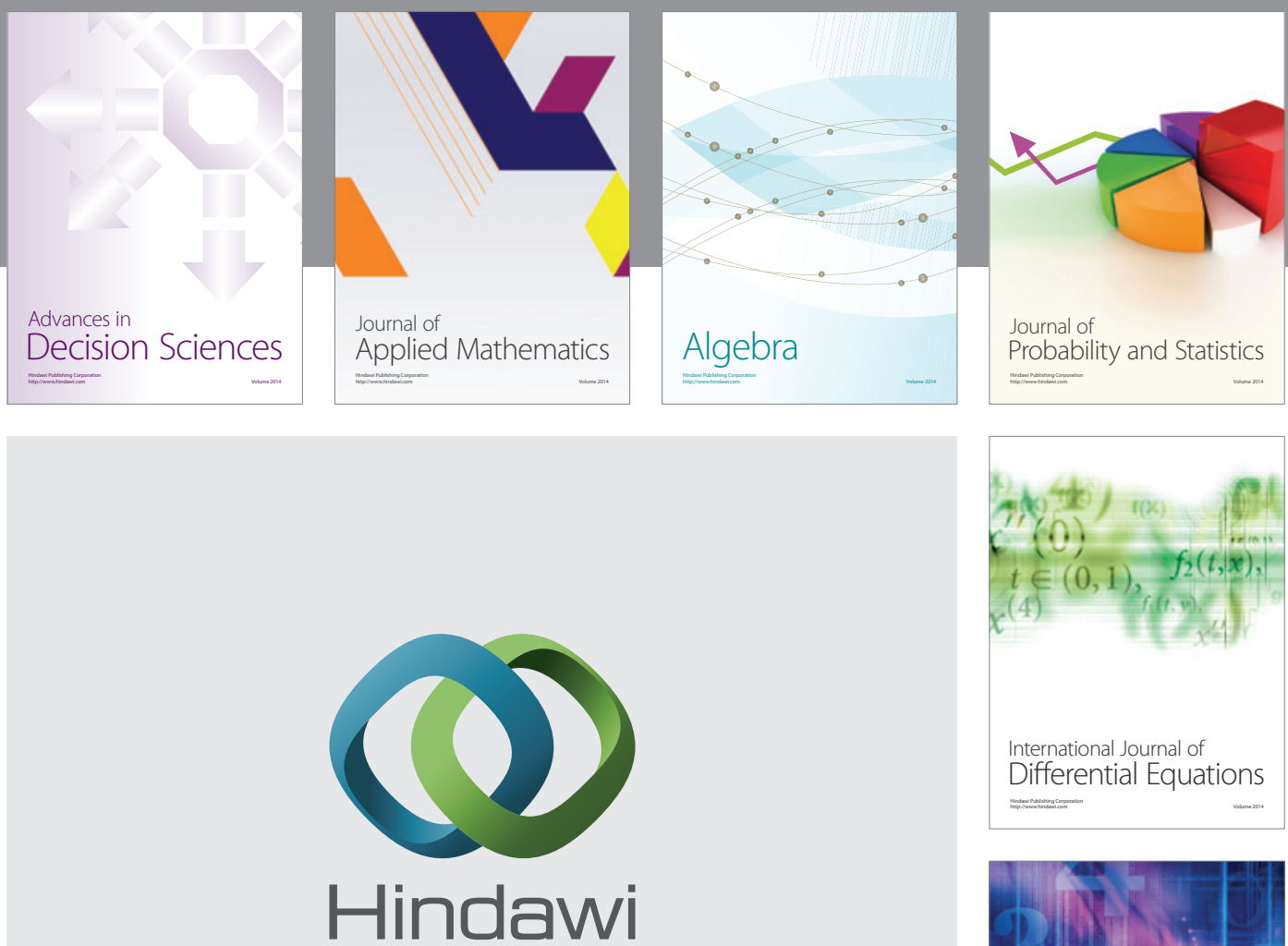

Submit your manuscripts at http://www.hindawi.com
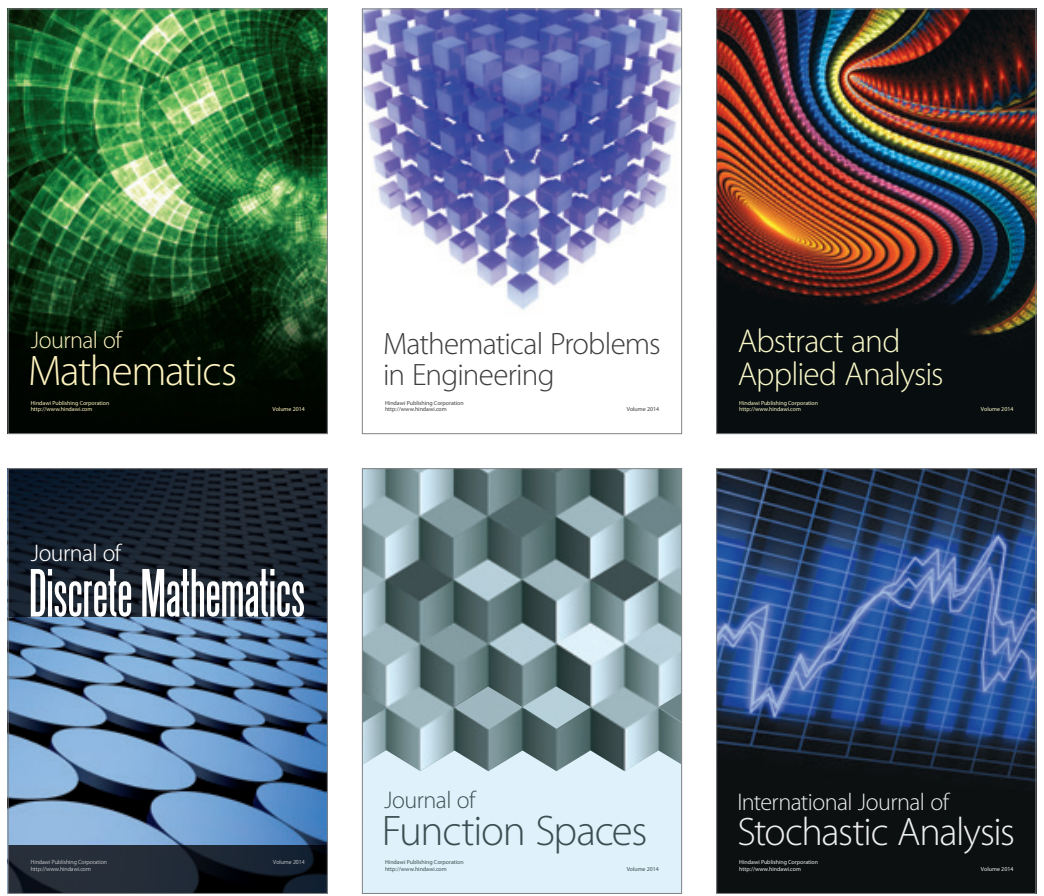

Journal of

Function Spaces

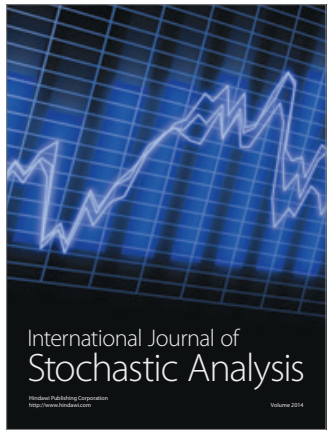

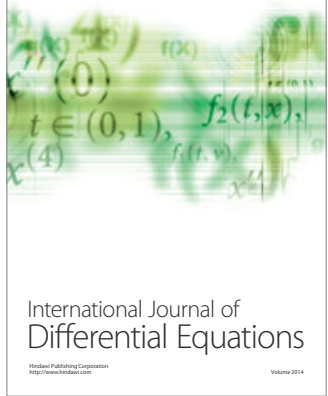
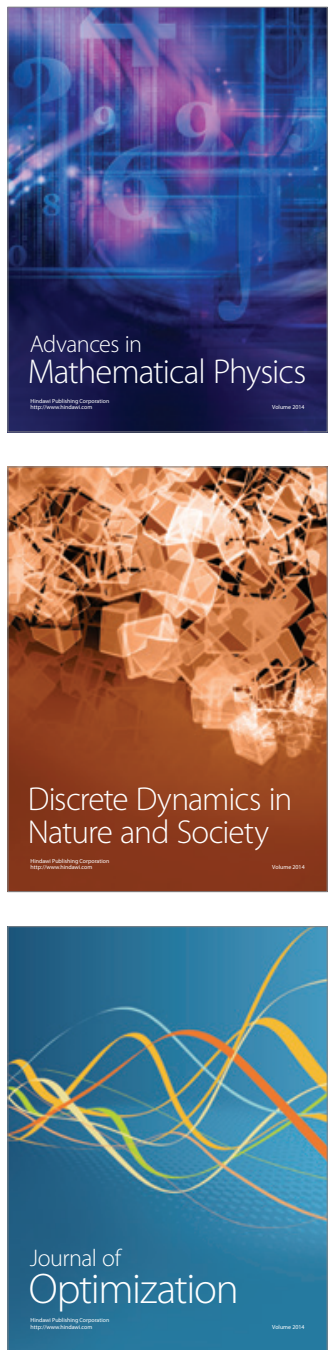